\title{
Factores psicosociales y síndrome de burnout en médicos de familia. México
}

\author{
Carolina Aranda-Beltrán ${ }^{1}$, Manuel Pando-Moreno ${ }^{1}$, Teresa Torres-López ${ }^{1}$, \\ José Salazar-Estrada ${ }^{1}$, Sergio Franco-Chávez ${ }^{1}$
}

Resumen

Objetivo: Determinar la presencia de los factores psicosociales laborales (sociodemográficos y laborales) y su relación con el síndrome de burnout en los médicos de familia que laboran en instituciones de salud. Materiales y Métodos: Para recabar la información necesaria en médicos de familia que laboraban en dos instituciones de salud para población derechohabiente en la zona metropolitana de Guadalajara, México, se utilizó dos cuestionarios: uno con variables sociodemográficas y laborales y el otro con la escala de Maslach Burnout Inventory (MBI-HSS) para evaluar la presencia del síndrome. Resultados: Participaron 197 médicos, 73 (37,1\%) mujeres y 124 (62,9\%) hombres. El 92,9\% contaba con la especialidad, los demás tenían la licenciatura; sin embargo, en su contrato de trabajo eran considerados como médicos de familia. La prevalencia de burnout en los médicos de familia fue 41,8\%. Tanto las asociaciones significativas como aquellas variables sociodemográficas y laborales que se comportaron como factor de riesgo se inclinaron hacia la dimensión "agotamiento emocional". Conclusiones: Aunque las variables en estudio fueron pocas, las que resultaron relacionadas con el desarrollo del síndrome fueron importantes como para ser vistas desde un nivel preventivo. Así mismo, se sugiere que quienes ya manifiestan signos y síntomas por este síndrome sean derivados para atención médica y/o psicológica y, si el caso lo requiere, brindarle incapacidad, con la finalidad de mejorar el bienestar y la salud del trabajador, así como la calidad de atención y de vida al derechohabiente.

Palabras clave Agotamiento profesional; médicos de familia; factores de riesgo; estrés psicológico; síndrome de adaptación general.

Psychosocial factors and burnout syndrome in family physicians. Mexico

\section{Abstract}

Objectives: To determine the presence of labor psychosocial factors (sociodemographic and labor factors) and their relationship with the burnout syndrome among family physicians. Materials and Methods: Two surveys were applied to family physicians who worked in two social security health institutions in the metropolitan area of Guadalajara, Mexico, in order to collect information on sociodemographic and labor variables and assess the presence of the syndrome with the Maslach Burnout Inventory Scale (MBI-HSS). Results: One hundred and ninety-seven physicians participated, $73(37,1 \%)$ women

1 Investigadores y Docentes del Departamento de Salud Pública, Universidad de Guadalajara. México. and 124 (62,9\%) men; 92,9\% had medical specialization and the rest had bachelor's degree but were considered family physicians according to their labor contract. The prevalence of burnout syndrome among family physicians was $41,8 \%$. Both, the significant associations and sociodemographic and labor variables risk factors tended towards the "emotional exhaustion" dimension. Conclusions: Even that few variables were introduced in this study, those related to the development of the syndrome were important to be considered in a preventive level. We suggest that people with the syndrome signs and symptoms be derived for medical and/or psychological attention, and if required, give them incapacity benefit, with the purpose of improving the worker's health and well-being as well as improving the social security worker's life and attention quality.

Keywords: Burnout, professional; physicians, family; risk factors; stress, psychological; general adaptation syndrome. 


\section{INTRODUCCIÓN}

En el año 2000, la Organización Mundial de la Salud consideraba que los trabajadores estaban expuestos a sufrir riesgos a la salud debido a las condiciones inseguras con las que laboran día a día. La presencia de estresores y la percepción que el trabajador tiene de que estos riesgos laborales junto con otros estresores extralaborales (personales, sociales, etc.) afectan su salud se conoce como "factores psicosociales laborales" $\left.{ }^{1-3}\right)$.

Según referencias emitidas por la OMS y la Organización Internacional del Trabajo (OIT), tanto los accidentes como las enfermedades laborales van en aumento. Así, para el año 2002 habría alrededor de 1,2 millones de muertes laborales, 250 millones de accidentes y 160 millones de enfermedades profesionales en el mundo, a diferencia de las presentadas en el 2001 con cifras de 1,1 millones de enfermedades y accidentes laborales, entre las cuales el estrés ocupaba un lugar importante $\left({ }^{4}\right)$.

En tal caso, los estudios sobre el estrés laboral crónico, también conocido como "síndrome de burnout o de desgaste profesional o asistencial", lo consideran (no en México) como una patología laboral debida al estrés continuo al que es sometido el médico durante su jornada laboral. Así, el estrés y el malestar acaban por instalarse de modo crónico, provocando trastornos psicosomáticos y de conducta, manifestaciones que nos alertan ante un problema de salud como lo es el síndrome de burnout.

Como ejemplos a los estudios realizados y a pesar del tiempo que se lleva estudiando este síndrome, ya desde Freudenberguer $\left(^{5}\right)$ y los estudios de Maslach $\left({ }^{6-8}\right)$; Fischer $\left({ }^{9}\right)$, Pines $\left({ }^{10}\right)$, Golembiewski $\left({ }^{11}\right)$, Price $\left({ }^{12}\right)$, Rodríguez $\left({ }^{13}\right)$, Gil-Monte $\left({ }^{1,2,14,15}\right)$, Capilla $\left({ }^{16}\right)$, Martínez $\left({ }^{17}\right)$, Cuevas $\left({ }^{18}\right)$, Aranda $\left({ }^{19,20}\right)$, en nuestro país, México, es poco conocido.

Se hace referencia de que la población más vulnerable a desarrollar este síndrome es la de los profesionales de la salud, principalmente personal de enfermería y médicos $\left({ }^{14,19,20-33}\right)$; menos frecuente, pero igual lo desarrollan, los trabajadores dedicados a los servicios sociales ${ }^{\left({ }^{3}\right)}$, docentes $\left({ }^{35-37}\right)$, secretarias $\left({ }^{38}\right)$, policías $\left({ }^{33,39}\right)$, estudiantes $\left({ }^{34}\right)$, etc.

Otras investigaciones señalan el efecto de las relaciones de poder, en donde mujeres de todas las edades padecen de discriminación en el empleo a causa del sexo y de su edad $\left({ }^{40-42}\right)$.

Por otro lado, los estudios relacionados con la presencia de factores psicosociales negativos en las áreas laborales giran desde los estudios sobre la búsqueda de factores varios (laborales y extralaborales) que puedan desarrollar la enfermedad $\left({ }^{43}\right)$, sobre las alteraciones psicosomáticas y sociales en trabajadores a quienes les han solicitado su renuncia $\left({ }^{44}\right)$, en hogares desorganizados $\left({ }^{45}\right)$, en personal que labora en servicios de atención en crisis y de servicio social $\left.{ }^{(33}\right)$, hasta en aquellos dedicados a los servicios de atención en emergencias y desastres $\left({ }^{46}\right)$.

Es así, como nace la inquietud de investigar y determinar la presencia de los factores psicosociales laborales (sociodemográficos y laborales) que el trabajador percibe como negativos y su relación con la presencia del síndrome de burnout en los médicos de familia que laboran para dos instituciones de salud para población derechohabiente en la zona metropolitana de Guadalajara, México.

\section{MATERIALES Y MÉTODOS}

El estudio es descriptivo y transversal. La población la constituyeron todos los médicos de familia que laboran en dos Instituciones de Salud para población derechohabiente por medio de un muestreo aleatorio estratificado, incluyendo a todos los que deseaban participar (mediante consentimiento informado); no se tomó en cuenta a quienes se encontraban ausentes en sus clínicas por encontrarse de permiso, de vacaciones o por 
cualquier otro motivo. Para tal caso, se visitaba nuevamente la clínica con el propósito de localizar al médico seleccionado según la muestra. Si después de 3 veces (intervalos de 15 días cada uno) aún no se encontraba el médico en su área de trabajo, se seleccionaba al sujeto inmediato posterior a él.

Para recabar la información necesaria, se utilizó dos cuestionarios. Uno era sobre datos generales y laborales con variables como nombre (opcional), edad, sexo, estado civil, escolaridad, número de hijos, tipo de contrato, puesto actual, turno, antigüedad en el puesto y en la institución. Para evaluar el síndrome de burnout, se utilizó la escala de valoración de "Maslach Burnout Inventory (MBI)" $\left({ }^{6-8}\right)$. Originalmente, este instrumento estaba formado por 25 ítems. Actualmente, consta de 22 reactivos, distribuidos en tres dimensiones: 1) agotamiento emocional (AE) formada por 9 ítems que describen sentimientos de estar agotados y abrumados (puntajes: alto $\geq 27$, medio 17 a 26 y bajo 0 a 16); 2) despersonalización (D), constituida por 5 reactivos, que describe una respuesta impersonal y falta de sentimientos (puntajes: alto $\geq 14$, medio 9 a 13 y bajo 0 a 8); y, 3) falta de realización personal y en el trabajo (FRP), con 8 ítems que describen sentimientos de falta de motivación (puntajes: alto $<30$, medio 36 a 31 y bajo 48 a 37), correspondiendo a las tres subescalas del MBI. Para las dimensiones "AE" y "D", altas puntuaciones corresponden a altos sentimientos de quemarse y para la de "FRP", bajas puntuaciones se relacionan a altos sentimientos de quemarse. Este instrumento es confiable, tanto en su validez factorial, concurrente y discriminante ${ }^{(1)}$.

Para el presente estudio, una persona que se encuentre en los niveles altos y medios será un sujeto con síndrome de burnout. Es importante señalar que esta escala (MBI-HSS) es la más conocida y las más utilizada, en diferentes poblaciones y actividades laborales.

Los análisis estadísticos fueron uno descriptivo y otro inferencial sobre la asociación y los factores de riesgo entre las variables psicosociales (sociodemográficas y laborales) con el síndrome de burnout, mediante el análisis estadístico de odds ratio (OR) cuando éste es mayor a uno, el intervalo de confianza (IC) no debía incluir la unidad; un chi cuadrado (con o sin corrección de Yates) era significativo con $p<0,05$. Para la realización de este análisis, se utilizó el paquete estadístico EPI INFO versión 6,04 .

\section{RESULTADOS}

La población en ambas instituciones consistió en 197 médicos, de los cuales $73(37,1 \%)$ eran mujeres y $124(62,9 \%)$ hombres. El médico más joven tenía 25 años de edad mientras que el más adulto 62 años, con un promedio de edad de 46,29 años. El grupo de edad más numeroso fue el de 45 a 49 años, $62(37,3 \%)$, seguido por el de 50 a 54 años, $36(21,7 \%)$, y por el de 40 a 44 , con $29(17,5 \%)$ sujetos.

En cuanto al estado civil, la mayoría era casado, 153 (79,7\%); el era soltero, viudo, divorciado. El 92,9\% tenía la especialidad; los demás tenían la licenciatura, sin embargo, en su contrato de trabajo eran considerados como médicos de familia; de ellos, $183(93,4 \%)$ eran de base, 4 eventuales y 9 residentes de la especialidad de medicina familiar. Ciento setenta y seis de los médicos se dedicaban a dar consulta en sus consultorios, sólo 19 lo hacían en el servicio de urgencias. Dos sujetos tenían apenas 6 meses de labor como médico familiar y uno rebasabe los 30 años, con un promedio de antigüedad de 16,47 años. Al agrupar la antigüedad en quinquenios, se observó que 54 $(27,4 \%)$ tenían entre 10 y 14 años de antigüedad, $48(24,4 \%)$ entre 15 y 19 años y $45(22,8 \%)$ entre 20 y 24 de labor. Cincuenta y un personas $(25,9 \%)$ tenían entre 10 y 14 años de laborar en ese puesto, $37(18,8 \%)$ entre 15 y 19 años y de 20 a 24 años $39(19,8 \%)$ médicos. La mayoría de los médicos laboraba en el turno matutino, $104(52,8 \%)$, seguido por el vespertino, 82 
$(41,6 \%)$. En cuanto a la carga horaria semanal, $107(54,6 \%)$ médicos refirieron trabajar 30 horas y $54(27,6 \%)$ dijeron tener un horario de 40 horas a la semana.

La prevalencia de burnout en los médicos de familia de ambas instituciones fue $41,8 \%$ (Tabla 1). Para cada una de las dimensiones, las prevalencias se presentaron de la siguiente manera: $31,2 \%$ de la población se encontraba agotada emocionalmente, $8,6 \%$ manifestaba síntomas de despersonalización, mientras que $14,3 \%$ estaba desmotivada para realizar su trabajo (Tabla 2).

Tabla 1. Prevalencia del síndrome de burnout según escala de Maslach Burnout inventory (MBI-HSS).

\begin{tabular}{lrr}
\hline No dimensiones quemadas $^{\circ}$ & $\mathrm{n}$ & \multicolumn{1}{c}{$\%$} \\
\hline Ninguna dimensión quemada & 114 & 58,2 \\
Una dimensión quemada & 62 & 31,6 \\
Dos dimensiones quemadas & 16 & 8,2 \\
Tres dimensiones quemadas & 4 & 2,0 \\
\hline
\end{tabular}

Fuente: directa.

Prevalencia de síndrome $=41,8 \%$.

En cuanto al análisis inferencial, éste demostró que las únicas asociaciones encontradas entre las variables sociodemográficas y laborales con el síndrome

Tabla 2. Prevalencia del síndrome de burnout por dimensiones obtenidas por el MBI-HSS.

\begin{tabular}{|c|c|c|c|c|c|c|}
\hline \multirow{3}{*}{$\begin{array}{l}\text { Nivel de } \\
\text { calificación }\end{array}$} & \multicolumn{6}{|c|}{$\begin{array}{l}\text { Dimensiones del síndrome de } \\
\text { burnout según el MBI }\end{array}$} \\
\hline & \multicolumn{2}{|c|}{$\begin{array}{l}\text { Agotamiento } \\
\text { emocional }\end{array}$} & \multicolumn{2}{|c|}{$\begin{array}{l}\text { Despersona- } \\
\text { lización }\end{array}$} & \multicolumn{2}{|c|}{$\begin{array}{c}\text { Falta de } \\
\text { realización } \\
\text { personal }\end{array}$} \\
\hline & $\mathrm{n}$ & $\%$ & $\mathrm{n}$ & $\%$ & $\mathrm{n}$ & $\%$ \\
\hline Alto & 26 & 13,3 & 4 & 2,0 & 13 & 6,6 \\
\hline Medio & 35 & 17,9 & 13 & 6,6 & 15 & 7,7 \\
\hline Bajo & 135 & 68,8 & 179 & 91,4 & 168 & 85,7 \\
\hline
\end{tabular}

Fuente: directa. de burnout se inclinaron hacia la dimensión agotamiento emocional, siendo la asociación con la institución, el estado civil, la escolaridad y el tiempo de laborar para esa institución (Tabla 3), y como factor de riesgo el estado civil (ser soltero) y la escolaridad (superior a la requerida por el puesto), con el desarrollo del síndrome de burnout y con la dimensión agotamiento emocional, específicamente (Tabla 4).

\section{DISCUSIÓN}

El hombre, además de trabajar, tiene otras rutas ineludibles: las superaciones personal, fraternal, filial, parental, conyugal, laboral, social, etc. Tiene que sortear satisfacciones y sufrimientos que le producen estrés y afectan su salud. El estrés produce respuestas a nivel individual, psicológicas y fisiológicas a un estímulo (estresor). Es un evento constante en el transcurso de toda la vida del trabajador, el cual forma parte de un obstáculo o reto que debe ser superado.

La entrada al síndrome de burnout es un distrés (estrés patológico) producido por un desequilibrio entre las expectativas y la realidad del trabajo, con manifestaciones psicológicas y físicas o la misma muerte, con repercusiones en la vida personal, familiar, social y en la institución donde labora. Es y debe ser reconocida en nuestro país, México, como una enfermedad laboral.

Las cifras de prevalencia encontradas, aunque no son tan altas como en otros estudios, son elevadas con respecto a otros países. Ello nos hace pensar que el síndrome está presente en esta población y que los factores sociodemográficos y laborales son causa; o al menos existe una relación significativa hacia el desarrollo del síndrome.

Es imprescindible, por un lado, el considerar que el punto de partida hacia el desarrollo del síndrome es a nivel organizacional; por tanto, se debe desarrollar programas de prevención 
Tabla 3. Únicas asociaciones significativas entre las variables sociodemográficas y laborales con el síndrome de burnout (MBI-HS).

\begin{tabular}{lcccc}
\hline \multirow{2}{*}{$\begin{array}{c}\text { Variables sociodemográficas } \\
\text { y laborales }\end{array}$} & \multicolumn{3}{c}{$\begin{array}{c}\text { Dimensiones del síndrome de burnout } \\
\text { valores con } p<0,05\end{array}$} \\
\cline { 2 - 5 } & AE & FRP & D & Toda la escala \\
\hline Institución & 0,0492 & $*$ & $*$ & $*$ \\
Sexo & $*$ & $*$ & $*$ & $*$ \\
Grupos de edad & $*$ & $*$ & $*$ & $*$ \\
Estado civil & 0,0222 & $*$ & $*$ & $*$ \\
Escolaridad & 0,0037 & $*$ & $*$ & 0,0117 \\
Puesto actual & $*$ & $*$ & $*$ & $*$ \\
Tipo de contrato & $*$ & $*$ & $*$ & $*$ \\
Antigüedad en la institución & 0,0336 & $*$ & $*$ & $*$ \\
Antigüedad en el puesto actual & $*$ & $*$ & $*$ & $*$ \\
Turno & $*$ & $*$ & $*$ & D: Despersonalización \\
\hline Fuente: directa & AE: Agotamiento emocional &
\end{tabular}

dirigidos a mejorar el ambiente y el clima laboral, como el crear programas de socialización, de apoyo, de trabajo en equipo, de planificación, de participación, de retroinformación, de crecimiento personal y de desarrollo organizacional. Por otro lado, los factores estresantes extralaborales también deben ser considerados con la misma importancia. Es necesario fomentar los valores personales y familiares, aprovechar cualquier momento de convivencia con la familia y con los compañeros de trabajo.

Tabla 4. Únicos factores de riesgo significativos entre las variables sociodemográficas y laborales con el síndrome de burnout (MBI-HSS).

\begin{tabular}{lcccc}
\hline \multirow{2}{*}{$\begin{array}{l}\text { Variables sociodemográficas } \\
\text { y laborales }\end{array}$} & \multicolumn{3}{c}{ Dimensiones del síndrome de burnout } \\
\cline { 2 - 5 } & $\mathrm{AE}$ & $\mathrm{FRP}$ & $\mathrm{D}$ & Toda la escala \\
\hline Institución & $*$ & $*$ & $*$ & $*$ \\
Sexo & $*$ & $*$ & $*$ & $*$ \\
Edad & $*$ & $*$ & $*$ & $*$ \\
Estado civil & $*$ & $*$ & $*$ & $\mathrm{OR}=2,17$ \\
Ser soltero & & & & $\mathrm{IC}=1,00-4,71$ \\
& & & & $p=0,0463$ \\
Escolaridad & $\mathrm{OR}=4.53$ & $*$ & $*$ & $\mathrm{OR}=7,97$ \\
Superior a la requerida por el puesto & $\mathrm{IC}=1,10-9,78$ & & & $\mathrm{IC}=1,51-56,25$ \\
& $p=0,0187$ & & & $p=0,0037$ \\
Puesto actual & $*$ & $*$ & $*$ & $*$ \\
Tipo de contrato & $*$ & $*$ & $*$ & $*$ \\
Antigüedad en la institución & $*$ & $*$ & $*$ & $*$ \\
Antigüedad en el puesto actual & $*$ & $*$ & $*$ & $*$ \\
Turno & $*$ & $*$ & $*$ & $*$ \\
\hline
\end{tabular}

Fuente: directa AE: Agotamiento emocional

D: Despersonalización

* Celdas con valores de OR no significativos FRP: Falta de realización personal y en el trabajo 
Las soluciones son preventivas y terapéuticas a nivel individual, grupal y organizacional. Entre las medidas preventivas tenemos la de evitar que la enfermedad o el daño llegue; si la enfermedad ya se instauró, habrá que controlarla, tratarla, pedir apoyo y evitar complicaciones $\left({ }^{1}\right)$. Cuanto más pronto se instaure las medidas preventivas, la evolución o curación serán una realidad.

\section{REFERENCIAS BIBLIOGRÁFICAS}

1. Gil-Monte PR, Peiró JM. Desgaste Psíquico en el Trabajo: El Síndrome de Quemarse. 1 ${ }^{\mathrm{a}}$ edición. Madrid: Síntesis; 1997.

2. Gil-Monte PR. Aproximaciones psicosociales y estudios diagnósticos sobre el síndrome de quemarse por el trabajo (Burnout). Psicología del Trabajo y de las Organizaciones. 2000;16(2):101-2.

3. Benavides G, Gimeneo D, Benach J, Martínez J, Jarque S, Berra A, et al. Descripción de los factores de riesgo psicosocial en cuatro empresas. Gaceta Sanitaria. 2002;16(3):222-9.

4. Organización Mundial de la Salud. Salud ocupacional: Éticamente correcta, económicamente adecuada. Ginebra: Organización Mundial de la Salud (OMS); 2000.

5. Freudenberger HJ. Staff burn-out. Journal of Social Issues. 1974;30(1):159-65.

6. Maslach C, Jackson SE. Maslach Burnout Inventory. Palo Alto (Ca): Consulting Psychologists Press; 1981.

7. Maslach C, Jackson SE. Burnout research in the social services: a critique. Special issues: Burnout among social workers. Journal of Social Service Research. 1985;10(1):95105.

8. Maslach C, Jackson SE, Leiter M. Maslach Burnout Inventory. Manual, $3^{\text {rd }}$. ed. Palo Alto (Ca): Consulting Psychologists Press; 1986.

9. Fischer HJ. (1983). A psychoanalytic view of burnout. En: Gil-Monte PR, Peiró SJM. Desgaste Psíquico en el Trabajo: El Síndrome de Quemarse. 1 ${ }^{\mathrm{a}}$ ed. Madrid: Síntesis; 1997.

10. Pines A, Aronson E. Career burnout: Causes and curses. En: Gil-Monte PR, Peiró SJM. Desgaste Psíquico en el Trabajo: El Síndrome de Quemarse. $1^{\mathrm{a}}$ ed. Madrid: Síntesis; 1997.

11. Golembiewski RT, Munzenrider RF, Carter D. Phases of progressive burnout and their work site covariantre: Critical issues in OD research and praxis. Journal of Applied Behavioral Science. 1983;19(4):461-81.

12. Price DM, Murphy PA. Staff burnout in the perspective of grief theory. Death Education. 1984;8(1):47-58.

13. Rodríguez MJ. Psicología Social de la Salud. Madrid: Síntesis; 1995.
14. Gil-Monte PR, Peiró JM, Valcárcel P. Influencia de las variables de carácter sociodemográfico sobre el Síndrome de burnout: Un estudio en una muestra de profesionales de enfermería. Revista de Psicología Social Aplicada. 1996;6(2):37-57.

15. Gil-Monte PR. El Síndrome de Quemarse por el trabajo (Síndrome de Burnout): Aproximaciones teóricas para su explicación y recomendaciones para la intervención. Psicología Organizacional. 2001;16:101-2.

16. Capilla PR. El Síndrome de burnout o de desgaste profesional. Revista Fundación Facultad de Medicina. 2000;58:1334.

17. Martínez de la Casa MA, Del Castillo CC, Magaña LE, Bru EI, Franco MA, Segura FA. Estudio sobre la prevalencia del burnout en los médicos del Área Sanitaria de Talavera de la Reina. Atención Primaria. 2003;32(6):343-8.

18. De las Cuevas CC. Burnout en los profesionales de la atención a las drogodependencias. Trastornos Adictivos. 2003;05(2):107-13.

19. Aranda BC. Apoyo social y Síndrome de quemarse en el trabajo "Burnout" en los médicos familiares de base del Instituto Mexicano del Seguro Social, Zona Metropolitana de Guadalajara. Tesis Doctoral en Ciencias de la Salud Pública. Universidad de Guadalajara, México, Enero 2004.

20. Aranda BC, Pando MM, Salazar EJG, Torres LTM, Aldrete RMG, Pérez RM. Síndrome de burnout y manifestaciones clínicas en los médicos familiares del Instituto Mexicano del Seguro Social, Guadalajara, México. Revista Cubana de Salud Pública. 2005;31(2). [en prensa]

21. Lavanco G. Burnout syndrome and type a behavior in nurses and teachers in Sicily. Psychological Reports. 1997;81:5238.

22. Martín MJ, Viñas J, Pérez J, Luque M, Soler IP. Burnout en personal de enfermería. Rev Psiquiatría Fac Med Barna. 1997;24(5):113-8.

23. Atance MJC. Aspectos epidemiológicos del Síndrome de burnout en personal sanitario. Revista Española de Salud Publica. 1997;71(3):293-303.

24. Pando MM, Bermúdez D, Aranda BC. El Síndrome del quemado en los trabajadores de la Salud. Revista Salud y Trabajo. 2000;1:12-5.

25. Cebriá J, Segura J, Corbella S, Sos P, Comas O, García $\mathrm{M}$, et al. Rasgos de personalidad en médicos de familia. Atención Primaria. 2001;27:459-68.

26. Gongalves EF, Aizpiri DJ, Barbado AJA, Cañones GPJ, Fernández CA, Rodríguez SJJ, De la Serna de Pedro I, Solla CJM. Síndrome de Burn-out en el médico general. Medicina General. 2002;43:278-83.

27. Prieto AL, Robles AE, Salazar MLM., Daniel VE. Burnout en médicos de atención primaria de la provincia de Cáceres. Aten Primaria. 2002;29(5):294-302.

28. Barría MJ. Síndrome de burnout en asistentes sociales del Servicio Nacional de Menores de la Región Metropolitana 
de Chile. Psiquiatria.com [serial en la Internet]. 2002 [citado 2005-06-03];6(4). Disponible en: http://www.psiquiatria.com

29. Martínez de la Casa MA, del Castillo CC, Magaña LE, Bru EI, Franco MA, Segura FA. Estudio sobre la prevalencia del burnout en los médicos del Área Sanitaria de Talavera de la Reina. Atención Primaria. 2003;32(6):343-8.

30. Álvarez AM, Arce ML, Barrios AE, Sánchez de Coll AR. Síndrome de burnout en médicos de hospitales públicos de la ciudad de Corrientes, Argentina. Revista de Posgrado de la Vía Cátedra de Medicina. 2005;141:27-30.

31. Román HJ. Estrés y burnout en profesionales de la salud de los niveles primario y secundario de atención. Revista Cubana de Salud Pública. 2003;29(2):103-10.

32. Albaladejo R, Villanueva R, Ortega P, Astasio P, Calle ME, Domínguez V. Síndrome de burnout en el personal de enfermería de un Hospital de Madrid. Revista Española de Salud Pública. 2004;(4)78:505-16.

33. Morales G, Gallego LM, Rotger D. La incidencia y relaciones de la ansiedad y el burnout en los profesionales de intervención en crisis y servicios sociales. Interpsiquis [serial en la Internet]. 2004 [citado 2005-06-05];(2004). Disponible en: http//www.cop.es

34. Aranda BC, Pando MM, Salazar EJG, Torres LTM, Aldrete RMG, Pérez RMB. Factores psicosociales laborales y síndrome de burnout en médicos del primer nivel de atención. Investigación en Salud. 2004;6(1):28-34.

35. Ferrando J, Pérez J. Un instrumento para medir quemazón profesional en los docentes: adaptación catalana del Maslach Burnout Inventory (MBI). Rev Psiquiatría Daf Med Barna. 1996;23(1):11-8.

36. Van-Horn JE, Schaufeli WB, Greenglass ER, Burke RJ. A Canadian Dutch comparison of teachers Burnout. Psychological Reports. 1997;81:371-82.

37. Aldrete RMG, Manuel PM, Aranda BC, Balcázar PN. Síndrome de burnout en maestros de educación básica, nivel primaria de Guadalajara. Investigación en Salud. 2003;5(1):11-6.

38. Manzano GG, Ramos CF. Profesionales con alto riesgo de padecer burnout: Enfermeras y Secretarias. Interpsiquis [serial en la Internet]. 2001 [citado 2004-09-27];(2). Disponible en: http//www.intersalud.es

39. Gil-Monte PR. Validez factorial de la adaptación al español del Maslach Burnout Inventory-General Survey. Salud Pública de México. 2002;44(2):33-40.
40. Bernard J, Meade K. Women come of age: perspectives on the lives of older women. Londres: Edward Arnold; 1993.

41. Itzin C, Newman J. Gender, culture and organizational change: putting theory into practice. Londres: Routledge; 1995.

42. Tyler M, Abbott P (1994). The commodification of sexuality: Sexuality labour markets. En: Arber Sara, Ginn Jay. Relación entre género y envejecimiento. $1^{\text {a }}$ ed. Madrid: Síntesis; 1996.

43. Cesarini J, Pagani JJ, Rodríguez AA, Wainszelbaum NR. El personal de salud, la actividad hospitalaria y la prevención del daño laboral. Boletín Científico, Asoc Med Municip de la CBA, Buenos Aires [serial en la Internet]. 2001 [citado 2005-06-07];6(25). Disponible en: http://www.medicosmunicipales.org.ar/bc0601.htm

44. Lozada MIO. Alteraciones psicosomáticas y sociales en trabajadores a quienes les han solicitado su renuncia. Revista Condiciones de Trabajo [serial en la Internet]. 1999 [citado 2004-10-11];XXIII(1). Disponible en: http:// www.stps.gob.mx

45. Guibert RW, Alonso RAP. Factores epidemiológicos y psicosociales que inciden en los intentos suicidas. Rev Cubana Med Gen Integr. 2001;17(2):155-63.

46. Condori L, Palacios A, Ego-Aguirre V. Impacto psicológico en el trabajo en emergencias y desastres en equipos de primera respuesta [monografía en la Internet]. Buenos Aires: monografias.com; 2005 [citado 2005-05-25]. Disponible en: http://www.monografias.com/trabajos 12/impsico/ impsico.shtml

Manuscrito recibido el 02 de setiembre de 2005 y aceptado para publicación el 30 de setiembre de 2005.

Correspondencia: Dra. Carolina Aranda Beltrán

Avenida Magisterio 1545 Casa 2

Col. Miraflores

C.P. 44270

Guadalajara, Jal, México.

Correo-e: caranda2000@yahoo.com.mx 